\title{
Dispositivo Vestível para Monitoramento de Pessoas Idosas
}

\author{
Elisângela Aparecida Dias \\ Universidade Positivo \\ Curitiba, Paraná \\ elisd2604@gmail.com \\ Caroline Mazetto Mendes \\ Universidade Positivo \\ Curitiba, Paraná \\ caroline.mendes@up.edu.br
}

\author{
Leonardo Sant'Anna Mariani \\ Universidade Positivo \\ Curitiba, Paraná \\ leonardomariani@msn.com \\ Verônica Isabela Quandt \\ Universidade Positivo \\ Curitiba, Paraná \\ veronica.quandt@up.edu.br
}

\begin{abstract}
Due to medical advances, life expectancy is getting longer and longer. Consequently the elderly population is growing and new challenges will be faced in the coming decades. In this context, this paper presents the development of a monitoring system for the elderly. The system includes a wearable device to detect falls, a panic button to be activated if the user feels unwell, and a speaker that beeps when medication is required. If the wearable device identifies a fall or detects panic button activation, an alert message is automatically sent to the mobile device of the responsible for the elderly. The expected result of this project is to efficiently monitor the elderly and to promote the quality of life and safety for elderly over 60 years.
\end{abstract}

\section{KEYWORDS}

Dispositivo Vestível, Monitoramento de Idosos, Segurança, Qualidade de Vida

\section{INTRODUÇÃO}

Com o avanço da medicina, a expectativa de vida está cada vez maior e, consequentemente, a população de idosos está aumentando. Segundo o Instituto Brasileiro de Geografia e Estatística - IBGE, em 2018, a proporção de pessoas com mais de 65 anos no Brasil era de 19,2 milhões $(9,2 \%)$ e a projeção para 2060 é que esse número aumente para 58,2 milhões (25,5\%) [1]. Pesquisas mostram que a queda entre idosos são frequentes e pode trazer consequências que alteram negativamente a qualidade dessas pessoas [2]. Além disso, uma parcela significativa de idosos apresenta dependência leve na realização das atividades básicas de vida diária [3].

Nesse contexto, fica caracterizada a necessidade de proporcionar melhor qualidade de vida e um envelhecimento ativo com independência funcional para essa importante parcela da população. Dentre diversas possibilidades, sistemas e tecnologias podem ser usados no cumprimento da tarefa, tendo em vista que isso deve ser meta dos poderes públicos e da sociedade em geral.

Tecnologias têm sido propostas para auxiliar o monitoramento de pessoas idosas. Cunha et al. [4] propuseram uma tecnologia pervasiva e móvel na prestação de cuidados para idosos. Sensores e atuadores são usados para aquisição da pressão arterial e batimentos cardíacos. O sistema inclui um aplicativo móvel e um software, que realizam o registro e manipulação de dados sobre os idosos, prestadores de serviços de saúde e emissão de relatórios, entre outras operações.
Mano et al. [5] desenvolveram um sistema chamado Health Smart Home. Consiste em um ambiente inteligente que permite o monitoramento e emissão de alertas para uma equipe médica ou um responsável. O ambiente é equipado com dispositivos tecnológicos tais como um dispositivo móvel e um relógio inteligente. A detecção de movimentos é realizada por meio de sensores, acelerômetro e giroscópio. Assim, os dispositivos tomam ações quando ocorre uma situação crítica, como por exemplo uma queda.

Carvalhido [6] desenvolveu uma metodologia de teste e certificação de componentes para ambientes assistidos. Os teste são realizados em um cenário em que há a presença de sensores. $\mathrm{O}$ sistema possui um módulo na nuvem que se comunica com os componentes e um aplicativo móvel. Assim, o sistema possibilita aos prestadores de cuidados de saúde acesso às informações dos sensores, que são captadas na casa dos idosos, fazem a interpretação desses e geram alertas se alguma situação de emergência ocorrer.

Nesse contexto, este trabalho apresenta o desenvolvimento de um sistema de monitoramento de pessoas idosas dentro de um ambiente assistido. O sistema inclui um dispositivo vestível e um aplicativo para dispositivos móveis. O dispositivo vestível contém sensores com os quais é possível detectar quedas. Além disso, ele contém um botão com a função pânico, que pode ser acionado pelo usuário caso ele se sinta mal. Ocorrendo uma queda ou acionamento do botão de pânico, o responsável pelo idoso é notificado sobre a necessidade de se tomar uma ação de auxílio.

\section{SOLUÇÃO PROPOSTA}

O dispositivo vestível é constituído de uma caixa pequena de plástico com um suporte para prender no cinto da calça. Ele é composto internamente por um microcontrolador ESP32, um acelerômetro ADXL345 e um buzzer. O microcontrolador faz o gerenciamento do acelerômetro configurado para monitorar quedas. O buzzer é usado para sinalizações.

Um botão com a função de pânico está presente na parte externa do dispositivo, podendo ser acionado caso o usuário se sinta mal. Ocorrendo queda ou acionamento do botão, uma mensagem é enviada ao servidor, por meio da placa integrada de wi-fi do microcontrolador. Se por algum motivo essa conexão for interrompida, o buzzer do dispositivo irá emitir dois beeps curtos continuamente até que ela seja restabelecida. A Fig. 1 mostra o diagrama de blocos do dispositivo.

O dispositivo conta ainda com um módulo carregador TP4056, um conversor regulador de tensão Step-up MT3608 e uma bateria 


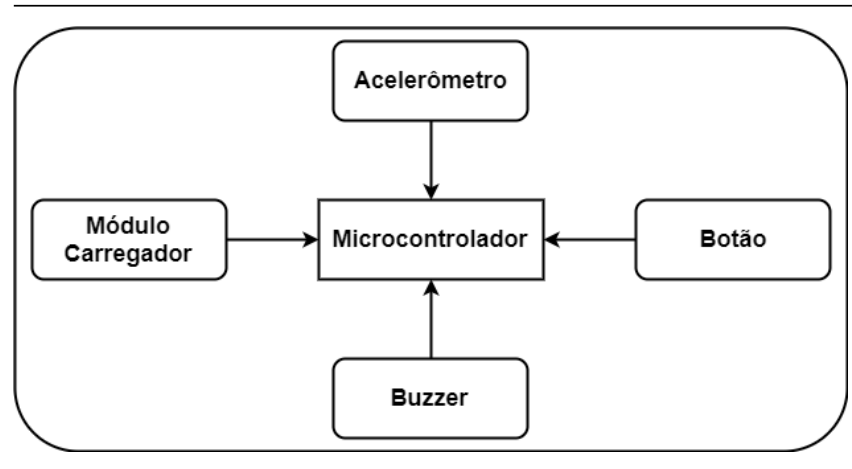

Figura 1: Diagrama de blocos do dispositivo vestível.

recarregável 3.6V 1400mAh. Com esses componentes é possível recarregar a bateria, a qual apresenta autonomia de aproximadamente 7 horas. Uma luz vermelha refletida na parte externa indica que o dispositivo está conectado na energia, enquanto uma luz azul indica o término do carregamento.

A Fig. 2 mostra os componentes internos inseridos dentro da caixa e também, mostra ele sendo carregado por meio de um cabo microUSB.

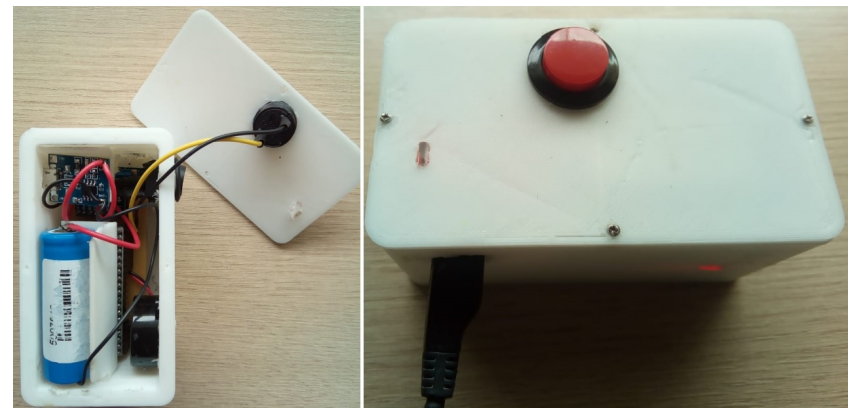

Figura 2: Protótipo do dispositivo vestível desenvolvido.

O aplicativo móvel foi desenvolvido para plataforma Android. Por meio desse aplicativo é realizado o cadastro do idoso. Podese também cadastrar informações sobre medicamentos, incluindo datas e horários da sua administração. O aplicativo também realiza a emissão de relatórios com a quantidade de quedas sofridas pelo idoso. Por fim, o sistema envia notificações para os celulares de responsáveis pelo idoso. A Fig. 3 mostra exemplos de notificações.

Os dados gerados pelo dispositivo móvel e dados cadastrados por meio do aplicativo são armazenados em um banco de dados na nuvem. Para essa tarefa foi usado o Firebase, o qual possui o recurso Firebase Cloud Messaging (FCM) para o envio de mensagens entre os componentes do sistema. Para o acionamento do buzzer foi usado o procolo MQTT (Message Queue Telemetry Transport), considerado padrão para comunicações IoT (Internet das Coisas).

\section{CONSIDERAÇÕES FINAIS}

Testes iniciais foram realizados. Para testar a identificação de quedas, o dispositivo vestível foi soltado a uma altura de 1 metro sobre uma almofada. Também, foi simulado o acionamento do botão de pânico,

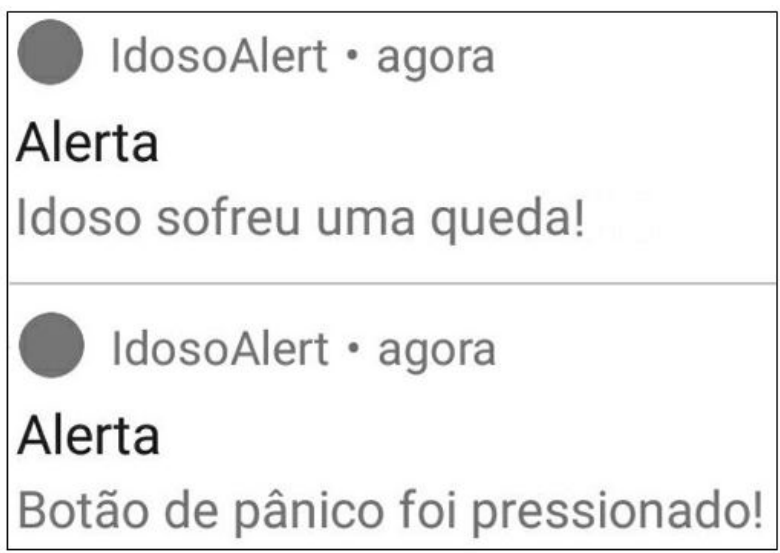

Figura 3: Notificação no celular do responsável.

o qual foi pressionado por 4 segundos. O acionamento do buzzer também foi testado, simulando o horário de uma medicação.

Os resultados mostraram que o sistema funciona corretamente, enviando mensagens de alerta para os responsáveis em caso de quedas e acionamento do botão de pânico. O sinal sonoro para administração de medicamentos também foi emitido pelo dispositivo, poucos segundos após a simulação.

Contudo, observou-se que o desempenho do sistema é dependente da qualidade de Internet disponível. Assim, foi constatada a necessidade de adicionar ao dispositivo vestível uma indicação para avisar o usuário se há conexão com a Internet. Também, é necessário realizar testes mais precisos da descarga da bateria.

Por fim, o dispositivo vestível será avaliado por pessoas idosas. Na primeira etapa da pesquisa, o participante deverá fazer uso do protótipo dentro da sua própria residência por pelo menos 7 dias consecutivos. Na segunda etapa, será aplicado um questionário com pessoas idosas, a fim de identificar a aceitação ou recusa das tecnologias de ambiente assistido.

\section{REFERÊNCIAS}

[1] IBGE. Projeção da população 2018: número de habitantes do país deve parar de crescer em 2047, 2018. URL https://agenciadenoticias.ibge.gov.br/agencia-salade-imprensa/2013-agencia-de-noticias/releases/21837-projecao-da-populacao2018-numero-de-habitantes-do-pais-deve-parar-de-crescer-em-2047. [Acessado em 14 de fevereiro de 2020].

[2] Adalgisa Peixoto Ribeiro, Edinilsa Ramos de Souza, Soraya Atie, Amaro Crispim de Souza, and Arthur Orlando Schilithz. A influência das quedas na qualidade de vida de idosos. Ciência \& Saúde Coletiva, 13:1265-1273, 2008.

[3] Maria Valdenize Melo da Silva and Alecsandra Ferreira Tomaz. Análise da qualidade de vida e capacidade funcional de idosos. TEMA-Revista Eletrônica de Ciências (ISSN 2175-9553), 18(28; 29), 2019.

[4] Carlos R Cunha, Vítor Mendonça, Elisabete Paulo Morais, and Joana Fernandes. Using pervasive and mobile computation in the provision of gerontological care in rural areas. Procedia computer science, 138:72-79, 2018.

[5] Leandro Yukio Mano, Marcio Maestralo Funes, Tiago Volpato, and José Rodrigues Torres Neto. Explorando tecnologias de iot no contexto de health smart home: uma abordagem para detecção de quedas em pessoas idosas. Fournal on Advances in Theoretical and Applied Informatics, 2(1):46-57, 2016.

[6] Bruno Miguel Carvalhido Lima. Teste e certificação de componentes para ecossistema de ambient assisted living. Master's thesis, Faculdade de Engenharia da Universidade do Porto, 2014. 